\title{
Variational Characterization of the Speed of Propagation of Fronts for the Nonlinear Diffusion Equation
}

\author{
R. D. Benguria and M. C. Depassier \\ Facultad de Física \\ P. Universidad Católica de Chile \\ Casilla 306, Santiago 22, Chile
}

\begin{abstract}
We give an integral variational characterization for the speed of fronts of the nonlinear diffusion equation $u_{t}=u_{x x}+f(u)$ with $f(0)=f(1)=0$, and $f>0$ in $(0,1)$, which permits, in principle, the calculation of the exact speed for arbitrary $f$.
\end{abstract}




\section{Introduction}

The problem of the asymptotic speed of propagation of the interface between an unstable and stable state has received much attention in connection with different problems of population growth, chemical reactions, pattern formation and others. We refer to [1] for a recent review and references. The best understood of such problems is that of the nonlinear reaction diffusion equation

$$
u_{t}=u_{x x}+f(u)
$$

with

$$
f(0)=f(1)=0, f^{\prime}(0)>0 \quad \text { and } \quad f>0 \quad \text { in } \quad(0,1)
$$

for which Aronson and Weinberger [AW] [2] have shown that any positive sufficiently localized initial condition $u(x, 0)$ evolves into a front that joins the stable state $u=1$ to $u=0$. The asymptotic speed at which the front propagates is the minimal speed $c^{*}$ for which there is a monotonic front joining $u=1$ to $u=0$. Moreover they show that the selected speed is bounded above and below by

$$
2 \sqrt{f^{\prime}(0)} \leq c^{*}<2 \sup \left\{\sqrt{\frac{f(u)}{u}} \mid u \in(0,1)\right\}
$$

and that the asymptotic selected front approaches the fixed point $u=0$ exponentially with slope

$$
m=-\frac{1}{2}\left(c^{*}+\sqrt{c^{* 2}-4 f^{\prime}(0)}\right) .
$$

The lower bound $c_{L}=2 \sqrt{f^{\prime}(0)}$ is that predicted from a physical argument, the linear marginal stability hypothesis [3]. For concave functions $f$, the upper and lower bounds coincide and the speed is exactly the linear value. However, the asymptotic speed of propagation can still be the linear value even when the upper and lower bounds do not coincide as explicit examples and a variational characterization [4] which provides improved upper bounds show. We have recently obtained an improved lower bound [5] on the speed of the front that enables one to decide when the selected speed is greater than the linear value case which is referred to as nonlinear marginal stability selection. There have been several reformulations of Aronson's 
and Weinberger's rigorous results for the nonlinear diffusion equation aiming to their heuristic extension to other higher order and pattern forming equations [6, 7, 8, 9]. None of these approaches however provide the means to calculate a priori the velocity of the fronts.

The purpose of the present work is to extend our previous result [5] to show an integral variational characterization of the speed of the fronts of equation (1) which enables, in principle, its exact calculation for arbitrary $f$. Our main result is the following

Theorem 1 (Variational Characterization of $c^{*}$ ) Let $f \in C^{1}(0,1)$ with $f(0)=f(1)=$ $0, f^{\prime}(0)>0$ and $f(u)>0$ for $u \in(0,1)$. Then

$$
c^{*}=\max \left\{2 \sqrt{f^{\prime}(0)}, J\right\}
$$

where

$$
J=\sup \{I(g) \mid g \in \mathcal{D}\}
$$

Here,

$$
I(g)=2 \frac{\int_{0}^{1} \sqrt{f g h} d u}{\int_{0}^{1} g d u}
$$

and $\mathcal{D}$ is the space of functions in $C^{1}(0,1)$ such that $g \geq 0, h \equiv-g^{\prime}>0$ in $(0,1), g(1)=0$,

and $\int_{0}^{1} g(u) d u<\infty$. Moreover, if $c^{*} \neq 2 \sqrt{f^{\prime}(0)}, J$ is attained at some $\hat{g} \in \mathcal{D}$, and $\hat{g}$ is unique up to a multiplicative constant.

In Section 2 we prove Theorem 1, and in Section 3 an example is given.

\section{Proof of the Variational Characterization}

We are interested in the calculation of the minimal speed for which equation (1) has a monotonic travelling front $u(x, t)=q(z)$ with $z=x-c t$ joining $u=1$ to $u=0$. Since the selected speed corresponds to that of a decreasing monotonic front, it is convenient to work in phase space. Calling $p(q)=-d q / d z$, where the minus sign is included so that $p$ is positive, we find that the monotonic fronts are solutions of

$$
p(q) \frac{d p}{d q}-c p(q)+f(q)=0
$$


with

$$
p(0)=0, \quad p(1)=0, \quad p>0 \quad \text { in }(0,1) .
$$

As shown by Aronson and Weinberger [2], the asymptotic speed of propagation of fronts of equation (1), $c^{*}$, is the minimum value $c$ for which there is a solution of (7) and (8). Aronson and Weinberger have proved that there is a (unique) $p$ satisfying (7) and (8) for $c=c^{*}$ (see [2], Section 4). Moreover, the solution $p$ is such that $p(q) \sim|m| q$ near $q=0$, where $|m|$ is the largest root of the equation

$$
x^{2}-c^{*} x+f^{\prime}(0)=0
$$

i.e.,

$$
|m|=\frac{1}{2}\left(c^{*}+\sqrt{c^{* 2}-4 f^{\prime}(0)}\right) .
$$

We find it convenient to introduce the parameter $\lambda$ defined as $\lambda=c^{*} /|m|$. In terms of $\lambda$ one can write

$$
c^{*}=\lambda \sqrt{\frac{f^{\prime}(0)}{\lambda-1}} \quad \text { and } \quad|m|=\sqrt{\frac{f^{\prime}(0)}{\lambda-1}} .
$$

It is straightforward to verify that whenever $1<\lambda<2$ the value of $|m|$ given by (10) corresponds to the largest root of (9) and therefore to the asymptotic slope at the origin of the selected front [10]. At $\lambda=2$ the speed $c^{*}$ attains the linear value $c_{L}$.

Proof of Theorem 1. The proof of the Theorem is done in two steps. First we show that

$$
c^{*} \geq \max \left\{2 \sqrt{f^{\prime}(0)}, J\right\},
$$

and then we show that either $c^{*}=2 \sqrt{f^{\prime}(0)}$ or $c^{*}=J$. From the results of Aronson and Weinberger $[\mathrm{AW}]$ it follows that $c^{*} \geq 2 \sqrt{f^{\prime}(0)}$ (see equation (2) above). Thus, to prove (11) we need only to show that

$$
c^{*} \geq I(g)
$$

for all $g \in \mathcal{D}$. This latter fact has been proven by us in [5]. We repeat here the argument for completeness. Let $g$ be any function in $\mathcal{D}$. Multiplying equation (7) by $g / p$ and integrating with respect to $q$ we find after integrating by parts,

$$
c^{*}=\frac{\int_{0}^{1}\left(h p+\frac{f(q)}{p} g\right) d q}{\int_{0}^{1} g(q) d q}
$$


However since $p, h, f$ and $g$ are positive, for every fixed $q$

$$
h p+\frac{f(q) g}{p} \geq 2 \sqrt{f g h}
$$

hence

$$
c^{*} \geq 2 \frac{\int_{0}^{1} \sqrt{f g h} d q}{\int_{0}^{1} g d q}
$$

which proves (11).

To finish the proof of the theorem notice that if $c^{*}=2 \sqrt{f^{\prime}(0)}$ we are done. Therefore, let's assume $c^{*} \neq 2 \sqrt{f^{\prime}(0)}$ (in fact, $c^{*}>2 \sqrt{f^{\prime}(0)}$ ). We will show that $c^{*}=J$ and that there exists $\hat{g} \in \mathcal{D}$ such that $c^{*}=I(\hat{g})$.

Let $p(q)$ be the positive solution of (7) satisfying (8). The existence of such a solution has been established by $[\mathrm{AW}]$. Moreover, $p(q) \sim|m| q$ near $q=0$.

In terms of $p$, let us define the function $v(q)$ as

$$
v(q)=\exp \left\{\int_{q_{0}}^{q} \frac{c^{*}}{p} d q\right\}
$$

for some fixed $0<q_{0}<1$. Since $p$ is continuous and strictly positive in $(0,1), v$ is $C^{1}$ in $(0,1)$. Moreover, $v$ satisfies

$$
\frac{v^{\prime}}{v}=\frac{c^{*}}{p} .
$$

Now choose

$$
\hat{g}=\frac{1}{v^{\prime}}
$$

From (15) and (17) it follows that

$$
\hat{g}(q)=\frac{p(q)}{c^{*}} \exp \left\{\int_{q}^{q_{0}} \frac{c^{*}}{p} d q\right\}
$$

Clearly, $\hat{g}(1)=0, \hat{g}(q)>0$, and since $p \in C^{1}(0,1), \hat{g} \in C^{1}(0,1)$. In order to show that $\hat{g} \in \mathcal{D}$, we must verify that $h \equiv-\hat{g}^{\prime}>0$ in $(0,1)$. It follows from (17) that

$$
\hat{g}^{\prime}=-\frac{v^{\prime \prime}}{v^{\prime 2}}
$$

and taking the derivative of (16) with respect to $q$ we have

$$
\frac{v^{\prime \prime}}{v}-\frac{v^{\prime 2}}{v^{2}}=-\frac{c}{p^{2}} p^{\prime}
$$


Using equations (7) and (16) to eliminate $p^{\prime}$ and $v^{\prime}$ in (19b) we find

$$
\frac{v^{\prime \prime}}{v}=\frac{c^{*} f}{p^{3}}
$$

Since $v, f$ and $p$ are positive, $v^{\prime \prime}>0$; hence, it follows from (19a) that $h=-\hat{g}^{\prime}>0$ in $(-0,1)$. Alternatively, it follows from (7) and (18) that

$$
h(q)=f(q) \exp \left\{\int_{q}^{q_{0}} \frac{c^{*}}{p} d q\right\} \geq 0 .
$$

Finally we must show that $\int_{0}^{1} \hat{g}(q) d q<\infty$. As we have seen, $\hat{g}$ is a continuous, positive and decreasing function in $(0,1)$. Hence, it is bounded away from the origin. Thus, to determine whether $\int_{0}^{1} \hat{g}(q) d q$ is finite we must study the behavior of $\hat{g}$ near $q=0$. Since we know that $p \sim|m| q$ near 0 , it follows from (16) that

$$
\frac{v^{\prime}}{v} \sim \frac{c^{*}}{|m|} \frac{1}{q}=\frac{\lambda}{q}
$$

which in turns implies that

$$
\hat{g} \sim \frac{1}{\lambda} \frac{1}{q^{\lambda-1}}
$$

near $q=0$. Therefore, if $\lambda<2$ (i.e., if $c^{*}>c_{L}$ ), we have $\int_{0}^{1} \hat{g}(q) d q<\infty$ and $g \in \mathcal{D}$.

Having verified that $\hat{g} \in \mathcal{D}$ we now show that for this choice of $\hat{g}$ the equality holds in (13). We must verify that

$$
h p+\frac{f \hat{g}}{p}=2 \sqrt{f \hat{g} h}
$$

or equivalently, that

$$
h p=\frac{f \hat{g}}{p} .
$$

This follows directly from the definition of $\hat{g}$, in fact from (19a) $h p \equiv-\hat{g}^{\prime} p=v^{\prime \prime} p / v^{\prime 2}$. Using equations (16), (20) and the definition (17) of $\hat{g}$ the result follows. Having shown that whenever $\lambda<2$ (i.e., whenever $c^{*}>c_{L}$ ) there exists a function $\hat{g} \in \mathcal{D}$ for which we obtain the exact speed we have proven (4).

Note: The uniqueness of the maximizer follows from the fact that we need to choose $\hat{g}$ in such a way that (13) is satisfied as an equality. Alternatively, we can prove uniqueness directly 
from the concavity of $I(g)$. From the homogeneity of $I(g)$ in $g$ we can restrict, without loss of generality, to $g$ 's such that $\int_{0}^{1} g(q) d q=1$. Assume $c^{*}>c_{L}$ and that there are two $g$ 's in $\mathcal{D}, g_{1}$ and $g_{2}$ say, such that $I\left(g_{1}\right)=I\left(g_{2}\right)=c^{*}$ (and $\left.\int g_{1}=\int g_{2}=1\right)$. Then, let $g=\alpha g_{1}+(1-\alpha) g_{2}$, whith $0<\alpha<1$. Clearly, $\int g=1$, and $h \equiv-g^{\prime}=\alpha h_{1}+(1-\alpha) h_{2}>0$. Therefore,

$$
I(g)=\int_{0}^{1} \sqrt{f\left(\alpha g_{1}+(1-\alpha) g_{2}\right)\left(\alpha h_{1}+(1-\alpha) h_{2}\right)} d q
$$

However, by the Cauchy-Schwarz inequality,

$$
\sqrt{\left(\alpha g_{1}+(1-\alpha) g_{2}\right)} \sqrt{\left(\alpha h_{1}+(1-\alpha) h_{2}\right.} \geq \alpha \sqrt{h_{1} g_{1}}+(1-\alpha) \sqrt{h_{2} g_{2}}
$$

with equality if and only if $g_{1}=\beta h_{1}$ and $g_{2}=\beta h_{2}$. Thus,

$$
c^{*} \geq I(g) \geq \alpha I\left(g_{1}\right)+(1-\alpha) I\left(g_{2}\right)=c^{*}
$$

which implies $h_{1} / g_{1}=h_{2} / g_{2}$, and therefore $g_{1} \equiv g_{2}$.

\section{Example}

In this section we illustrate the results by applying it to the exactly solvable case $f(u)=$ $u(1-u)(1+a u)$ for which it is known that

$$
c^{*}= \begin{cases}\sqrt{\frac{2}{a}}+\sqrt{\frac{a}{2}} & \text { if } a>2 \\ 2 \sqrt{f^{\prime}(0)}=2 & \text { if } a<2\end{cases}
$$

The idea is to combine the variational characterization contained in Theorem 1, which given a trial function yields lower bounds for $c^{*}$, with the variational characterization of Hadeler and Rothe [4], which provides upper bounds on $c^{*}$.

For a given $a>0$, let us take

$$
g(q)=\frac{(1-q)^{\lambda+1}}{q^{\lambda-1}} \quad \text { with } \lambda=1+\frac{2}{a} .
$$

Then

$$
h=-g^{\prime}=\frac{(1-q)^{\lambda}}{q^{\lambda}}(\lambda-1)(1+a q)
$$


and [1]

$$
\int_{0}^{1} g(q) d q=\frac{\Gamma(\lambda+2) \Gamma(2-\lambda)}{\Gamma(4)} \quad \text { if } \quad \lambda<2
$$

i.e., if $a>2$.

We obtain

$$
\int_{0}^{1} \sqrt{f g h} d q=\sqrt{\lambda-1}\left(\int_{0}^{1} \frac{(1-q)^{\lambda-1}}{q^{\lambda-1}} d q+a \int_{0}^{1}(1-q)^{\lambda+1} q^{2-\lambda} d q\right)
$$

and therefore

$$
I(g)=2 \sqrt{\lambda-1}\left(1+a \frac{\Gamma(3-\lambda)}{\Gamma(2-\lambda)} \frac{\Gamma(4)}{\Gamma(5)}\right)
$$

Using the definition of $\lambda$ and $\Gamma(z+1)=z \Gamma(z)$ we obtain

$$
I(g)=\sqrt{\frac{2}{a}}+\sqrt{\frac{a}{2}}
$$

for $a>2$. Therefore, from Theorem 1 we have that

$$
c^{*} \geq \begin{cases}\sqrt{\frac{2}{a}}+\sqrt{\frac{a}{2}} & \text { if } a>2 \\ 2 \sqrt{f^{\prime}(0)}=2 & \text { if } a<2\end{cases}
$$

To prove the reversed inequality we use Hadeler and Rothe's variational characterization 四:

$$
c^{*} \leq \sup _{u}\left\{\dot{\rho}(u)+\frac{f(u)}{\rho(u)}\right\}
$$

for any $\rho \in C^{1}(0,1)$ such that $\rho(0)=0$ and $\rho(u)>0$ in $(0,1)$.

For $a \geq 2$ choose $\rho(u)=\sqrt{a / 2} u(1-u)$. Then $\dot{\rho}+(f / \rho)=\sqrt{a / 2}+\sqrt{2 / a}$. For $a \leq 2$ choose $\rho=u(1-u)$. Hence, $\dot{\rho}+(f / \rho)=2+(a-2) u$ and $\sup _{0 \leq u \leq 1}(\dot{\rho}+(f / \rho))=2$. Therefore, from Hadeler's and Rothe's bound we get

$$
c^{*} \leq \begin{cases}\sqrt{\frac{2}{a}}+\sqrt{\frac{a}{2}} & \text { if } a>2 \\ 2 \sqrt{f^{\prime}(0)}=2 & \text { if } a<2\end{cases}
$$

which combined with our lower bound gives the desired result. 


\section{Conclusion}

We have given a variational characterization of the minimal speed for which the nonlinear diffusion equation has monotonic fronts. As [AW] have shown this is the asymptotic speed of propagation of a sufficiently localized positive initial condition $u(x, 0)$.

The variational principle we have derived here can also be used to study the dependence of $c$ on the parameters of $f$. Monotonicity properties can be immediatly derived. Derivatives of $c$ with respect to parameters of $f$ can be obtained using the Feynman-Hellmann formula.

\section{Acknowledgments}

This work was partially supported by Fondecyt project 193-0559.

\section{References}

[1] Cross, M. C., Hohenberg P. H.: Pattern Formation Outside of Equilibrium. Rev. Mod. Phys. 65, 851-1112 (1993)

[2] Aronson D. G., Weinberger H. F.: Multidimensional Nonlinear Diffusion Arising in Population Genetics. Adv. Math. 30, 33-76 (1978)

[3] Kolmogorov A. N., Petrovskii I. G. and Piskunov N. S.: A Study of the Diffusion Equation with Increase in the Amount of Substance, and its applications to a Biological Problem. In Seleceted Works of A. N. Kolmogorov. V. M. Tikhomirov (Ed.), Kluwer Academic Publishers, 1991

[4] Hadeler K. P., Rothe F.: Travelling Fronts in Nonlinear Diffusion Equations. J. Math. Biol 2, 251-263 (1975)

[5] Benguria R. D., Depassier M. C.: Validity of the Linear Speed Selection Mechanism for Fronts of the Nonlinear Diffusion Equation. Phys. Rev. Lett. 73, 2272-2275 (1994) 
[6] Dee G. T., Langer J. S.: Propagating Pattern Selection. Phys. Rev. Lett. 50, 383-386 $(1983)$

[7] Ben-Jacob E., Brand H., Dee G. T., Kramer L. and Langer J. S.: Pattern Propagation in Nonlinear Dissipative Systems. Physica D 14, 348-364 (1985)

[8] van Saarloos W.: Front Propagation into Unstable States. II Linear versus Nonlinear Marginal Stability and Rate of Convergence. Phys. Rev. A 39, 6367-6390 (1989)

[9] Paquette, G. C., Chen, L. Y., Goldenfeld, N. and Oono Y.: Structural Stability and Renormalization Group for Propagating Fronts. Phys. Rev. Lett. 72, 76-79 (1994)

[10] Benguria R. D., Depassier M. C.: Exact Front Solutions for the Nonlinear Diffusion Equation with Quintic Nonlinearities. To appear in Phys. Rev. E.

[11] Abramowitz M. and Stegun, I. A., (Eds.), Handbook of Mathematical Functions, National Bureau of Standards Applied Mathematics Series 55, U.S. Government Printing Office, Washington D.C., 1964 